\title{
Optically understanding the dependence of catalysis kinetics on work function of nanocatalyst
}

\author{
HENAM SYLVIA DEVI, THIYAM DAVID SINGH and HENAM PREMANANDA SINGH* \\ Department of Basic Sciences and Humanities, National Institute of Technology, Imphal 795001, India
}

MS received 14 October 2015; accepted 27 June 2016

\begin{abstract}
In this work, biogenic $\mathrm{ZnO}$ and $\mathrm{CuO}$ nanoparticles were fabricated by adopting a complete green aqueous approach utilizing Centella asiatica plant extract. The phytochemicals involved in tailoring the nanoscopic metal oxide particles were characterized vividly. Both of the as-synthesized metal oxide particles are spherical and have a narrow size distribution with an average diameter of about $7 \mathrm{~nm}$ as measured via HRTEM. Interestingly, X-ray diffraction (XRD) pattern of $\mathrm{ZnO}$ nanoparticles displays the exposure of its (100) hexagonal facet. The ensuing $\mathrm{ZnO}$ and $\mathrm{CuO}$ nanoscale particles briskly enhanced the reduction of methylene blue, which was otherwise very slow. The reaction follows first-order kinetics and the rate constants follow the order $k_{\text {uncat }}<k_{\mathrm{ZnO} \text { NPs }}<k_{\mathrm{CuO} \text { NPs }}$. The higher catalytic performance of copper oxide nanoparticles than zinc oxide nanoparticles is succinctly established on the basis of their difference in work function values optically.
\end{abstract}

Keywords. Green synthesis; Centella asiatica; metal oxide nanocatalyst; methylene blue; work function.

\section{Introduction}

Nanotechnology is widely contemplated to become the next technological revolution following on from the first Industrial Revolution that began around 1760. Development and applications of well-defined nanodimensional structures are promising in numerous areas such as optics, drug-gene delivery, mechanics, biomedical sciences, chemical industry, electronics, space industries, energy science, catalysis, optoelectronic devices, photoelectrochemical applications and nonlinear optical devices [1-5]. Recently, much attention has been paid to nanostructures of transition metal oxides because of their unique characteristic features such as efficient charge separation, excellent catalytic activity, high photosensitivity, imaging tendency, magnetic and optical properties [6-9]. Catalysis remains at the heart of countless chemical techniques from the academic point of view to industries. About $80 \%$ of the processes in chemical industries depend on efficient working of catalysts [10]. An area of catalysis that is developing briskly is nanocatalysis. But understanding the factors that influence activity and selectivity of catalysts is still a key issue among the scientific community.

Although there is an array of chemical, physical and biological techniques that are utilized to tailor nanoscale materials, fabrication involving safe, nontoxic, less sophisticated and environmentally benign methods is preponderant. This leads to the critical role of micro-organisms and plant extracts in the production of nanoparticles (NPs). Such biogenic synthesis is necessary not only because of its reduced impact on environment, but also it can be employed for the

*Author for correspondence (henam_boynao@yahoo.co.in) production of large quantities of contamination-free NPs, which exhibit a well-defined size and morphology [11,12]. Various plant species may serve as a natural reservoir for the generation of myriad of metal and metal oxide NPs [2,1315]. However, only few works are available in the literature for the synthesis of NPs using Centella asiatica plant extract and are limited particularly to gold and silver NPs [16,17].

C. asiatica is a perennial herbaceous creeping plant generally grown widespread as wild. Further, various bioactive ingredients reported in $C$. asiatica were alkaloids, tannins, flavonoids, steroids, terpenoids, carbohydrates, phenolic compounds, vitamin $\mathrm{K}$ and amino acids. This herb is edible, has medicinal properties such as neuroprotective activity, anti-oxidant effect, anti-fever, healing of wounds and skin treatment, and even possesses anti-tumour ability [16-19].

Considering all these views, herein we demonstrate a simple, high-atom-economy and cost-effective green aqueous route for the preparation of biogenic zinc oxide $(\mathrm{ZnO})$ and copper oxide $(\mathrm{CuO})$ particles in the nanoregime using $C$. asiatica plant extract as effective heterogeneous nanocatalysts for reduction of organic pollutants such as dyes. Methylene blue (MB) was employed as a model dye for this experiment and we optically implicated the influence of metal oxide nanocatalyst work function, a fundamental aspect of catalyst, on catalytic activity rather than the geometrical factors.

\section{Materials and methods}

\subsection{Materials}

All the chemical reagents used were of AR grade. Cupric acetate was procured from Alice Chemicals Pvt. Ltd. Cochin 
and zinc nitrate hexahydrate from S.D. Fine Chem. Ltd. Fresh $C$. asiatica plants were collected from local area of Imphal, Manipur, India, and dried at room temperature. A syringe filter was obtained from Sartorius, while MB and sodium borohydride were purchased from Merck and Spectrochem, respectively. All the chemicals were used as such without any further purification and their solutions were prepared in double distilled water (DDW).

\subsection{Synthesis of active $\mathrm{ZnO}$ and $\mathrm{CuO}$ nanocatalysts}

Preparations of nanoscale $\mathrm{ZnO}$ and $\mathrm{CuO}$ particles were accomplished by reacting $\mathrm{Zn}\left(\mathrm{NO}_{3}\right)_{2}$ and $\mathrm{Cu}\left(\mathrm{CH}_{3} \mathrm{COO}\right)_{2}$ solution with $C$. asiatica plant extract. The plant extract was prepared by stirring $500 \mathrm{mg}$ of plant material in $20 \mathrm{ml}$ of DDW and stirred continuously for $30 \mathrm{~min}$ at room temperature. Properly cleaned, aerially dried $C$. asiatica plant was utilized for the fabrication protocol. This extract to be used as a reaction medium was then separated from $C$. asiatica solid material using a syringe filter. Now, $250 \mu \mathrm{l}$ of $3 \%(\mathrm{w} / \mathrm{v})$ each metal salt solution was added in $9.75 \mathrm{ml}$ aliquot of filtered C. asiatica plant extract and further stirred for $30 \mathrm{~min}$. The overall reaction mixture was maintained at $50^{\circ} \mathrm{C}$. Throughout the preparation, water was utilized as the environmentally benign solvent. The ensuing $\mathrm{ZnO}$ and $\mathrm{CuO}$ NPs were collected via ultracentrifugation, washed thoroughly with DDW and finally dispersed in it.

\subsection{Characterization of the synthesized nanoscale particles}

2.3a Ultraviolet-vis spectrum: All UV-vis spectra were monitored on a Shimadzu-1601 UV-vis spectrophotometer fitted with a constant-temperature cell holder. The temperature of the cell holder can be maintained by circulating water around it by a water circulator from Haake Instruments. The absorption spectra of the tailored NPs were recorded by measuring the aqueous dispersion of the NPs and scanned in the range of $200-900 \mathrm{~nm}$.

2.3b X-ray diffraction analysis: X-ray diffraction (XRD) of the particles was carried out on a Phillip X'pert XRD instrument. An adequate amount of dried powder samples was used to carry out the diffraction experiment.

2.3c Scanning electron microscopy: The measurements were performed with a SEI quanta 250 model scanning electron microscopy (SEM) instrument. Dried powder samples of the so-synthesized nanoscale metal oxides particles were utilized for this purpose.

2.3d Transmission electron microscopy and selected area electron diffraction micrograph: Transmission electron microscopy (TEM) and selected area electron diffraction (SAED) images of the particles were recorded using a JEM2100 transmission electron microscope operating at 60$200 \mathrm{KV}$. After preparation, the $\mathrm{ZnO}$ and $\mathrm{CuO}$ NPs were centrifuged at $10000 \mathrm{rpm}$ for $10 \mathrm{~min}$ and re-dispersed in DDW after sonicating for 4-5 min. A drop of dilute aqueous dispersion was put on a copper grid and the grid was allowed to dry under ambient conditions. TEM and SAED pictures of the nanoscale particles were obtained after complete drying of the grids.

2.3e Dynamic light scattering and zeta potential ( $\zeta$-potential) measurement: Dynamic light scattering (DLS) and $\zeta$-potential of the synthesized $\mathrm{ZnO}$ and $\mathrm{CuO}$ NPs were monitored using a Zetasizer Ver. 7.11 instrument from Malvern Instruments Ltd. The hydrodynamic diameter $(d)$ of the NPs was calculated from the diffusion of the particles using the Stokes-Einstein equation. Both the measurements were performed in aqueous medium at room temperature. Thoroughly washed particles were utilized for these purposes.

2.3f Fourier transformed infrared spectroscopy: Fourier transformed infrared (FT-IR) spectra of the NPs and plant extract were recorded using an FT-IR-Perkin Elmer-UATRTwo instrument. The particles after fabrication were centrifuged at $10000 \mathrm{rpm}$, collected and then washed with DDW three times. These particles were finally re-dispersed in DDW and their liquid IR spectra were monitored at room temperature. The samples were scanned in the range $650-4000 \mathrm{~cm}^{-1}$.

\subsection{Kinetics analysis of $M B$ reduction}

Reduction of $\mathrm{MB}$ by $\mathrm{NaBH}_{4}$ in aqueous medium was studied spectrophotometrically at $662 \mathrm{~nm}$, a prominent characteristic peak of MB at room temperature. To a mixture containing 20 $\mu \mathrm{l}$ of $\mathrm{MB}\left(1 \times 10^{-3} \mathrm{M}\right)$ and $50 \mu \mathrm{l}$ of $\mathrm{NaBH}_{4}$ solution $(0.1 \mathrm{M})$, $1 \mathrm{ml}$ of $7.5 \times 10^{-3} \%$ (w/v) aqueous dispersion of $\mathrm{ZnO}$ (or $\mathrm{CuO}$ ) NPs was added in a $3.5 \mathrm{ml}$ capacity quartz cuvette. The total volume of the mixture was maintained at $3 \mathrm{ml}$ by adding $1.93 \mathrm{ml}$ of DDW. The reactions were studied spectrophotometrically at a constant temperature of $25^{\circ} \mathrm{C}$ by circulating water from a thermostated water bath around the cuvette. The blue colour of the reaction mixtures gradually faded, indicating that reduction of MB had occurred. In a similar fashion, for the uncatalysed reaction, instead of metal oxide NPs $1 \mathrm{ml}$ of DDW was added to maintain the total volume of the reaction mixture. The reaction was followed by monitoring the time-dependent absorbance in a UV-vis spectrophotometer at $662 \mathrm{~nm}$ and the fall in absorbance with respect to time was recorded.

\section{Results and discussion}

To study the effect of catalyst work function on catalytic reaction rate, we have successfully fabricated nanodimensional $\mathrm{ZnO}$ and $\mathrm{CuO}$ particles by adopting an environmentally benign green synthesis route in aqueous medium using C. asiatica plant extract. The appearance of pale yellow and brownish colour upon addition of zinc and copper salt, 


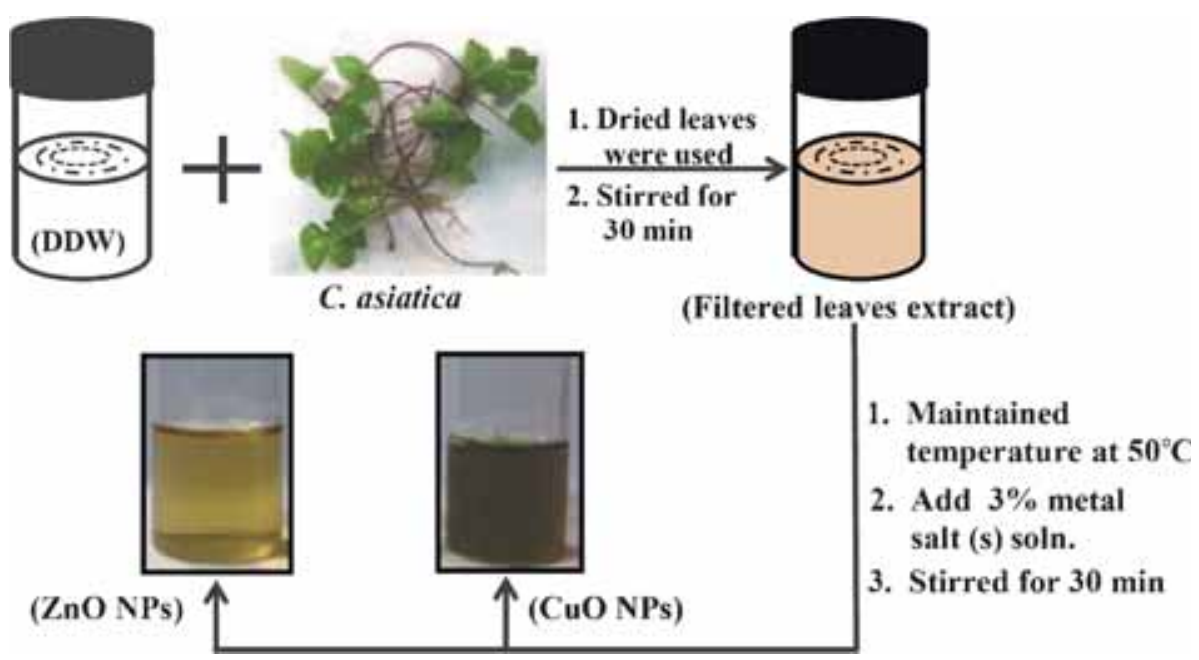

Scheme 1. Synthesis methodology of $\mathrm{ZnO}$ and $\mathrm{CuO}$ NPs.

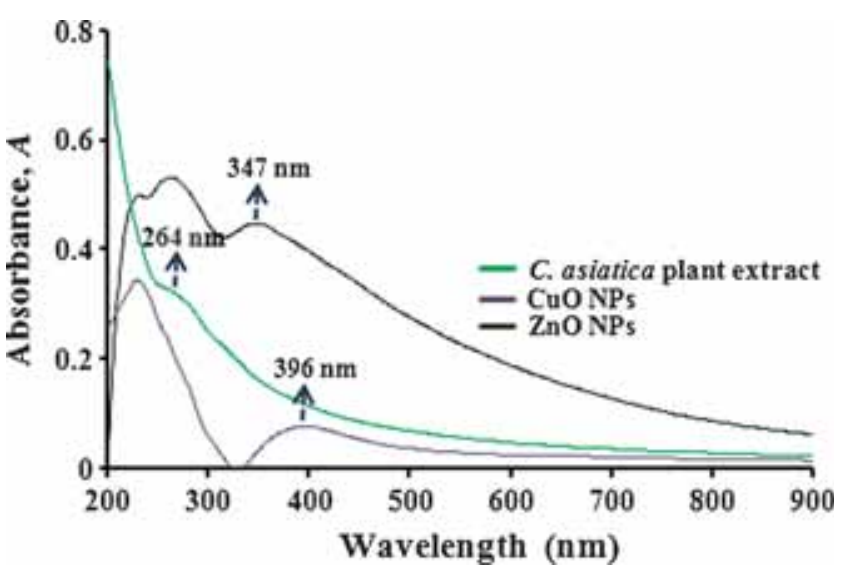

Figure 1. UV-vis absorption spectrum of C. asiatica plant extract and metal oxide NPs.

respectively, to light yellowish colour of plant extract indicates the formation of NPs. A schematic representation of the overall synthesis methodology is illustrated in scheme 1 .

Our approach excludes the usage of organic solvents, surfactants, harsh environment and strong reducing and stabilizing agents, unlike many conventional techniques. Figure 1 displays the UV-vis absorption spectra of $\mathrm{ZnO}$ and $\mathrm{CuO}$ particles exhibiting prominent surface plasmon characteristic peaks centred at 347 and $386 \mathrm{~nm}$, respectively, which confirm directly the formation of nanoscale particles [20,21]. The aqueous dispersions of these NPs are clear and remain unsettled for more that $72 \mathrm{~h}$ as we noticed, revealing that no aggregation of particles has taken place. Thus, phytochemicals present in the plant extract served a dual propose by acting as both reducing as well as stabilizing agent. The phytochemicals existing in $C$. asiatica plant extract are surface active and might have enough potential to wrap efficiently around the particles, thereby restricting their growth, and provide excellent robustness against agglomeration [22,23].
SEM images of $\mathrm{ZnO}$ and $\mathrm{CuO}$ NPs presented in figure 2 further support the formation of particles. The images reveal high density of the synthesized nanoscopic particles but determinations of the size and morphology of the particles were difficult within the measured resolution. However, a closer inspection displayed deposition of ultrafine particles over another aggregate formed. This might be due to the complete drying of the samples involved during SEM measurements.

TEM analyses were carried out to validate UV-vis spectroscopy and SEM experiments. The average diameters of the two different metal oxide NPs are equivalent and are about $7 \mathrm{~nm}$ as confirmed by our TEM results displayed in figure 3a. The so-obtained $\mathrm{ZnO}$ and $\mathrm{CuO}$ NPs are almost spherical and have a narrow size distribution. Figure $3 \mathrm{~b}$ displays a typical SAED pattern of $\mathrm{ZnO}$ and $\mathrm{CuO}$ NPs. The patterns exhibit dotted ring structures, which supplement crystalline behaviour of these particles; $\zeta$-potential values as highlighted in figure $3 \mathrm{c}$ of the two different metal oxide NPs in aqueous medium are also almost identical and it is about $-29.5 \mathrm{mV}$.

However, from the size distribution histograms obtained via DLS measurement, the average sizes of $\mathrm{ZnO}$ and $\mathrm{CuO}$ NPs were 10 and $9 \mathrm{~nm}$, respectively, as presented in figure 4 . These slight variations in the average diameters as monitored by DLS and HRTEM can be ascertained to the fact that in TEM we utilized dried form of the synthesized particles but in DLS the size of particles was estimated in aqueous dispersion, which provides the hydrodynamic diameter of the particles.

Nonetheless, powder XRD graphs in $2 \theta$ range of $30-70^{\circ}$ further substantiate crystalline nature of the tailored metal oxide NPs, shown in figure 5. ZnO NPs show only two diffraction peaks at 31 and $34.98^{\circ}$, which may be assigned, respectively, to (100) and (002) of hexagonal phase structure [24] as highlighted in figure 5a. The ratio of the intensity of 

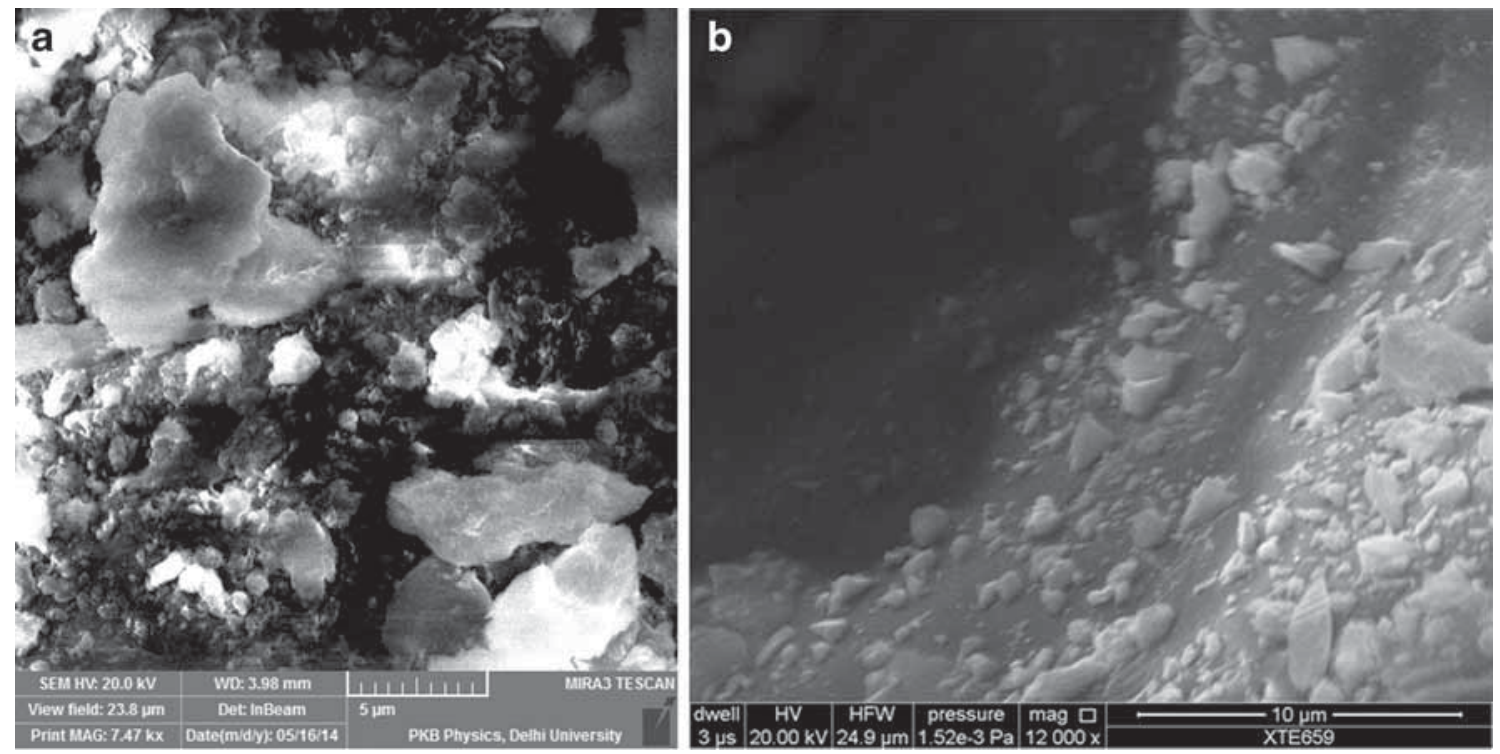

Figure 2. Typical SEM images of (a) $\mathrm{ZnO}$ and (b) $\mathrm{CuO}$ NPs.

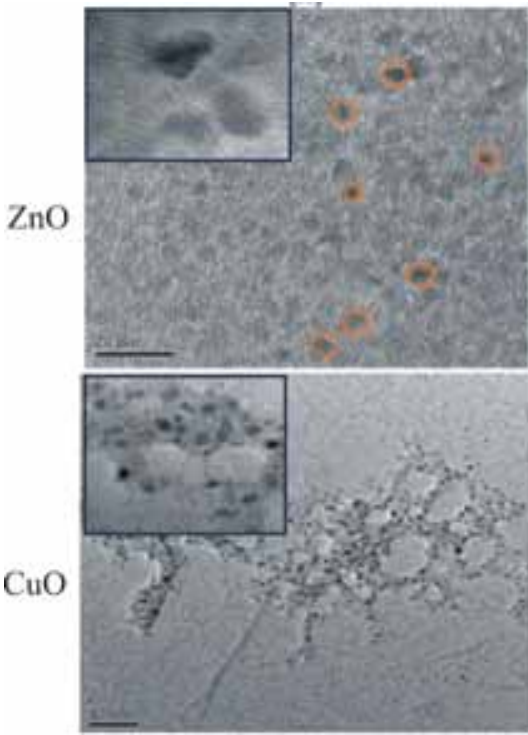

(a)

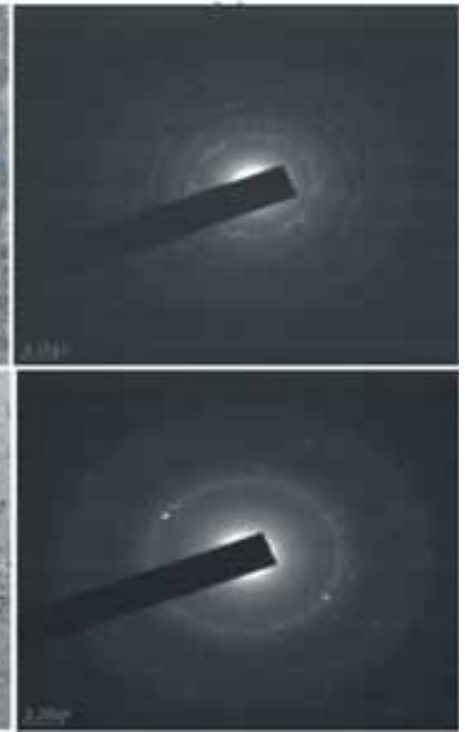

(b)
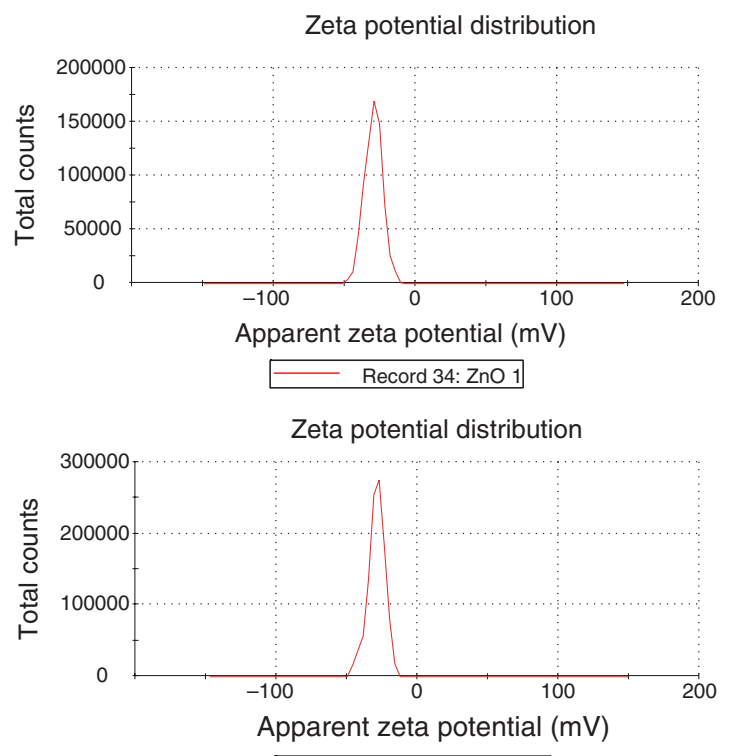

(c)

Record 33: CuOa 1

Figure 3. (a) TEM micrographs, (b) SAED patterns and (c) $\zeta$-potential of $\mathrm{ZnO}$ and $\mathrm{CuO}$ NPs.

(100) to (002) hexagonal facets of the as-synthesized $\mathrm{ZnO}$ NPs is obviously larger than that of conventional bulk $\mathrm{ZnO}$, which consequently implies the exposure of its (100) crystal plane as in the case of (100) and (111) surfaces exposure of nanocrystalline $\mathrm{CeO}_{2}$ generated by Deori et al [25]. Such surface exposure of a particular crystal facet plays a crucial part in altering the properties of a material.

Unlike $\mathrm{ZnO}$ NPs, $\mathrm{CuO}$ nanoscale particles exhibit three characteristic peaks at $36.8,49.98$ and $52.14^{\circ}$ corresponding to monoclinic crystal planes $(-111),(-202)$ and $(020)$, respectively, as shown in figure $5 \mathrm{~b}$ [26]. However, the peaks are not very intense. Interestingly, no extra diffraction peaks of impurities or metal salts were noticed, suggesting that both the synthesized metal oxide particles were essentially pure.

Further, figure 6a illustrates FT-IR spectrum of the $C$. asiatica plant extract with a sharp characteristic peak of $\mathrm{C}-\mathrm{N}$ stretching vibration of aliphatic amines at 1052 $\mathrm{cm}^{-1}$ and $\mathrm{C}-\mathrm{N}$ stretching for aromatic amines at $1376 \mathrm{~cm}^{-1}$. At $1642 \mathrm{~cm}^{-1}, \mathrm{C}=\mathrm{O}$ stretching vibration in carbonyl compounds, which may be characterized by the presence of high content of terpenoids and flavonoids, can be noticed vividly. The band in the region $3100-3400 \mathrm{~cm}^{-1}$ is due to $\mathrm{OH}$ stretching vibration and due to the wide variety of hydrogen bonding between $\mathrm{OH}$ groups, revealing the inclusion of 


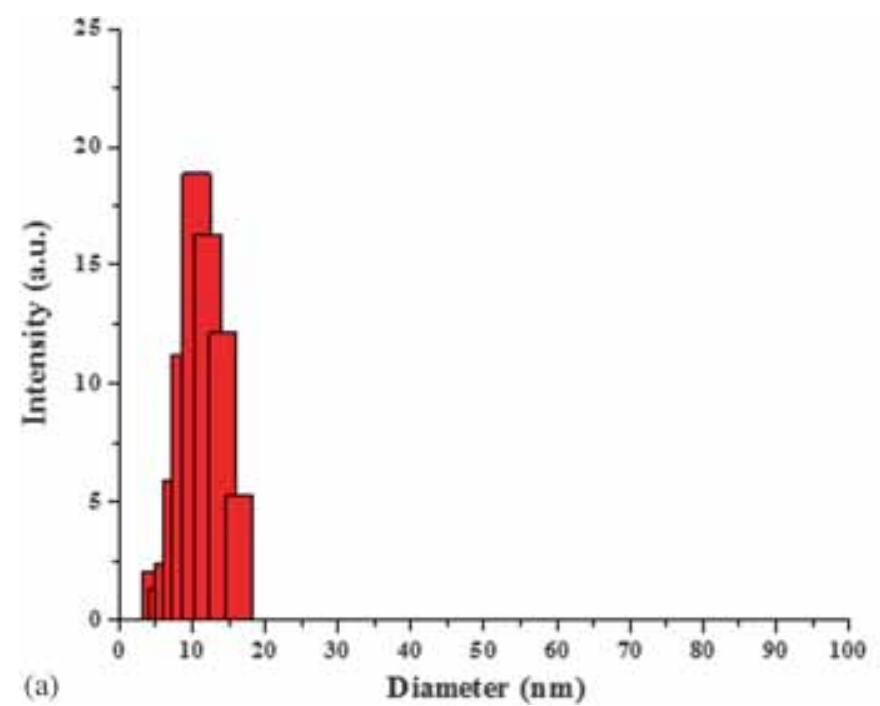

Figure 4. DLS patterns of the fabricated (a) $\mathrm{ZnO}$ and (b) $\mathrm{CuO}$ NPs.

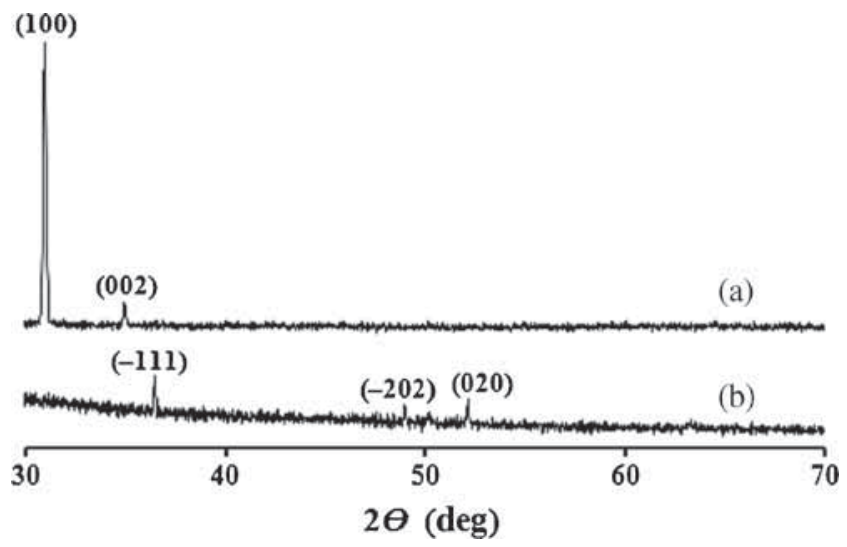

Figure 5. XRD patterns of nanoscopic (a) $\mathrm{ZnO}$ and (b) $\mathrm{CuO}$ particles.

polyphenolic compounds. The peak located at around 2352 $\mathrm{cm}^{-1}$ was attributed to $\mathrm{N}-\mathrm{H}$ stretching vibrations and the broad intense band around $3402 \mathrm{~cm}^{-1}$ in the spectrum can be assigned to the $\mathrm{N}-\mathrm{H}$ stretching arising from the peptide linkages present in the protein of the extract [17,27]. These bands originated from the functional groups of the phytochemicals incorporated with the plant extract and are also predominantly observed in cases of metal oxide NPs also as highlighted in figure $6 \mathrm{~b}$ and $\mathrm{c}$. The overall observations confirm the presence of polyphenols, including tannin, flavonoids and protein or amino acid along with the metal oxide NPs. Thus, inclusion of such phytochemicals might be the key determinant of the size and morphology of the resultant NPs.

The metal oxide NPs thus prepared by the abovementioned procedure were analysed for their catalytic potential in the reduction of MB to its colourless leuco form by $\mathrm{NaBH}_{4}$ at room temperature. The progress of the reactions between $\mathrm{MB}$ and $\mathrm{NaBH}_{4}$ in the presence and absence of nanocatalysts was monitored spectrophotometrically by
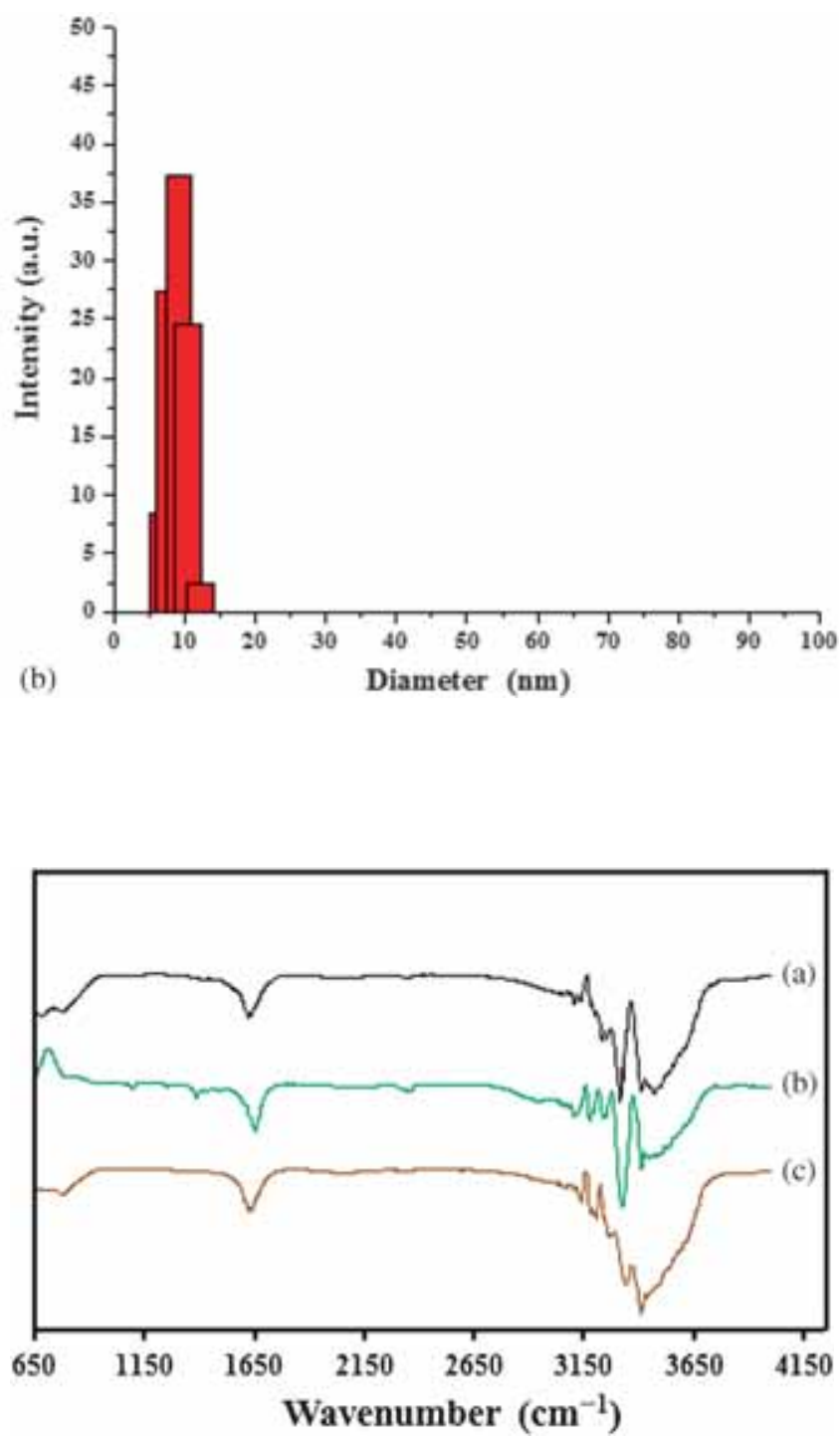

Figure 6. FT-IR spectra of (a) plant extract, (b) $\mathrm{CuO}$ and (c) $\mathrm{ZnO}$ NPs.

observing the disappearance in absorbance at a wavelength of $662 \mathrm{~nm}$ as depicted in figure 7 .

Figure 7a shows that the reduction of $\mathrm{MB}$ with $\mathrm{NaBH}_{4}$ in the absence of catalyst occurs at an extremely slow rate under the given time constraint even in the presence of a strong reducing agent, $\mathrm{NaBH}_{4}$, indicating that the reaction has large kinetic energy barrier. However, the presence of metal oxide NPs remarkably enhances the reduction reaction, especially in case of $\mathrm{CuO}$ NPs. The catalytic efficacies of the different nanoscopic metal oxide particles are different as displayed in figure $7 \mathrm{~b}$ and c. From the graph we observed that the concentration of $\mathrm{MB}$ falls briskly in the presence of CuO NPs within $1 \mathrm{~min}\left(\Delta A_{1} \min =1.846\right)$ as compared with ZnO NPs $\left(\Delta A_{12} \min =1.865\right)$ in $12 \mathrm{~min}$, while there is negligible reduction of $\mathrm{MB}$ up to $60 \mathrm{~min}$ for the uncatalysed reaction $\left(\Delta A_{60 \mathrm{~min}}=0.146\right) ; \Delta A$ is the change in absorbance. The reaction of $\mathrm{MB}$ with $\mathrm{NaBH}_{4}$ using the plant extract instead of NPs exhibits almost the same pattern as in 


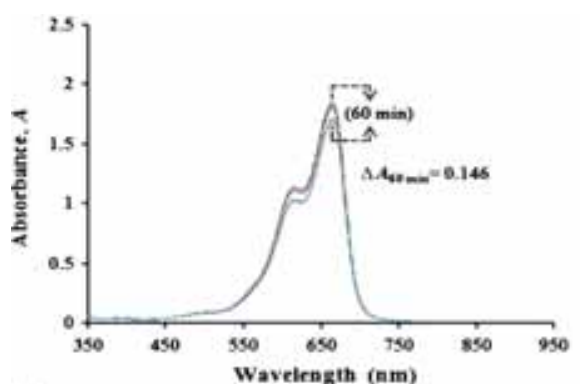

(a)

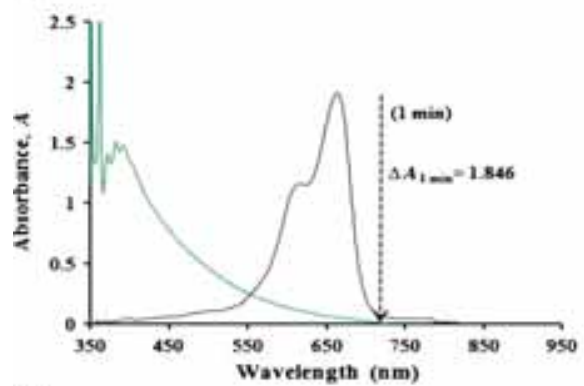

(c)

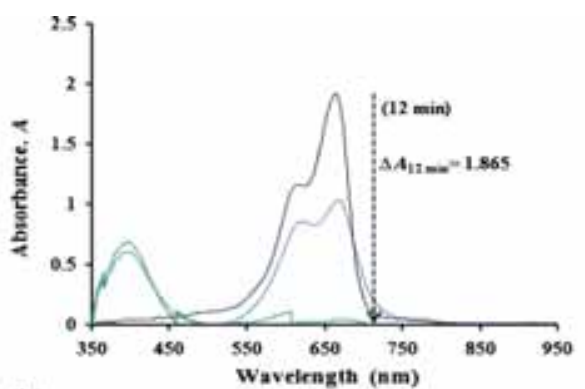

(b)

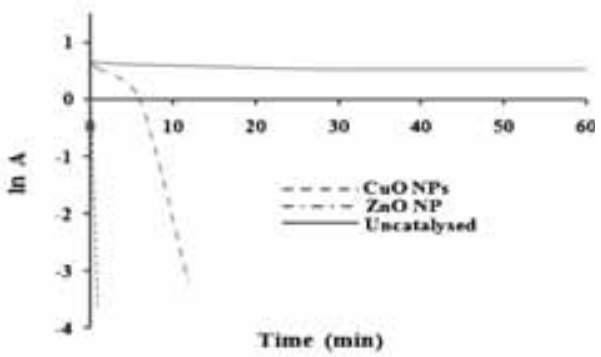

(d)

Figure 7. Plot of absorbance vs. wavelength for chemical reduction of $\mathrm{MB}$ with $\mathrm{NaBH}_{4}$ in the (a) absence of catalyst, (b) presence of ZnO NPs and (c) presence of CuO NPs; and (d) their kinetics plot.

case of the absence of nanocatalysts (identical to figure 7a), which indicated that $\mathrm{ZnO}$ and $\mathrm{CuO}$ NPs act as effective heterogeneous catalysts and not the phytochemicals incorporated with the plant extract. Moreover, no induction period was observed during the course of reactions. During the reaction the concentration of $\mathrm{NaBH}_{4}$ largely exceeds the concentration of $\mathrm{MB}$ and may be considered to remain constant throughout the reaction. It will be a reasonable assumption that the reduction reaction follows pseudo-first-order kinetics with respect to $\mathrm{MB}$.

The rate of the reaction can be expressed as

$$
\mathrm{d} C / \mathrm{d} t=k C,
$$

where $C$ is the concentration of MB at any instant of time $t$ and $k$ is the rate constant. In terms of absorbance $A$, the above equation can also be written as

$$
\ln \left(C_{t} / C_{0}\right)=\ln \left(A_{t} / A_{0}\right)=k t \text {. }
$$

The rate constants of the catalysed and uncatalysed reactions determined from the slope of natural logarithm of the absorbance $v s$. time curve are presented in figure $7 \mathrm{~d}$ and are summarized in table 1.

The rate constant $k$ for the reduction of MB follows the order $k_{\text {uncat }}<k_{\mathrm{ZnO} N P s}<k_{\mathrm{CuO} \text { NPs. }}$. The high catalytic reaction rate may be attributed to small size of the synthesized NPs, allowing more atoms on the surface than in the bulk. As a consequence they have large surface to volume ratio, which in turn enhances the number of active sites, facilitating more reactants molecules to interact and proceed to the reaction simultaneously. Even though the surface-exposed (100) facet of $\mathrm{ZnO}$ NPs might have facilitated the reaction intrinsically, it exhibits lesser kinetic rate compared with $\mathrm{CuO}$ NPs that do not lay bare crystal facets. Generally, surface exposure enhances the fraction of surface atoms and
Table 1. Rate constant $k$ for uncatalysed and catalysed reduction of $\mathrm{MB}$ with $\mathrm{NaBH}_{4}$ in aqueous medium at $25^{\circ} \mathrm{C}$.

\begin{tabular}{cc}
$\begin{array}{c}\text { Type of metal oxide } \\
\text { nanocatalysts }\end{array}$ & $\begin{array}{c}\text { Rate constant } k \\
\left(\mathrm{~min}^{-1}\right)\end{array}$ \\
\hline Uncatalysed & $2.0 \times 10^{-3}$ \\
ZnO NPs & $3.11 \times 10^{-1}$ \\
$\mathrm{CuONPs}$ & 4.261 \\
\hline
\end{tabular}

hence the performance of a particle as a catalyst significantly depends on the number of surface atoms for coordinating with reactant species. Indeed, it seems that besides the most commonly accepted catalyst's surface quality there is a more complex factor of the catalysts that substantially propels the catalytic efficiency predominantly. The variation in the above catalytic reduction rates for the two different metal oxide NPs may be figured out on the basis of catalyst work function values. During catalysis, work function of a catalyst (metal, metal oxide, alloy and semiconductor) is expected to play a pivotal role.

From the expressions $[27,28]$

$$
-\mathrm{d} I=\left(8 \pi^{3} / 3 h^{2}\right)\left|\mu_{\operatorname{lm}}\right|^{2}(I / c) h v_{\operatorname{lm}}(\mathrm{NC} / 1000) \mathrm{d} l
$$

and

$$
A=\left\{8 \pi^{3} N / 3 h c(1000)\right\} v_{\operatorname{lm}}\left|\mu_{\operatorname{lm}}\right|^{2}
$$

it is concluded that

$$
-\mathrm{d} I=A I C \mathrm{~d} l
$$

which on integration yields

$$
I=I_{0} \mathrm{e}^{-A C l},
$$


where $I$ is the intensity or energy associated with the radiation, $A$ is the integral absorption coefficient, $C$ is the molar concentration and $l$ is the path length. Expression (1) shows that the energy is exponentially related to absorbance and this is in accordance with our observation of work function (or energy) with absorbance. This result is further supported by the exponential relationship of the rate of particle catalyst reaction with the average catalyst work function observed by Vayenas et al [29] in 1990. They elucidated the dependence of catalytic rate on catalyst work function via electrochemical study. According to them the rate of catalytic reactions depends exponentially on catalyst work function by the following relation:

$$
\ln \left(r / r_{0}\right)=\alpha e\left(\Phi-\Phi^{*}\right) / k_{\mathrm{B}} T,
$$

where $e \Phi^{*}$ is the threshold work function value and $r_{0}$ is the regular catalytic rate; $\alpha$ and $\Phi^{*}$ are reaction- and catalystspecific constants, respectively.

From equations (1) and (3) it can be concluded that the rate of change of absorbance (which is the reaction rate) varies exponentially with the catalyst work function.

But work function of the catalyst exhibits size- and morphology-dependent property. It is higher for small particles as compared with their conventional bulk counterpart. Such shifting of work function from bulk to small metallic spheres has a classical interpretation based on classical picture and Coulomb potentials of spherical geometry and is provided by the following expression [30]:

$$
\varphi=\varphi^{*}+\left(e^{2} / 4 \pi \varepsilon_{0}\right)\{[(q+1) / r]-(5 / 8 r)\},
$$

where $\varphi^{*}$ is the work function at the surface, which is quite identical to the bulk value, $r$ is particle radius and $q$ is the initial charges carried by the particle. For single-charge particles $(q=0)$ the particle work function equation (4) reduces to (in the unit of eV)

$$
\varphi=\varphi^{*}+[1.08(\mathrm{eV}) / D(\mathrm{~nm})],
$$

where $D$ is the diameter of a spherical particle in the unit of 'nm'. Considering equations (4) and (5), it can be anticipated that since the average sizes and zeta potential values of $\mathrm{ZnO}$ and $\mathrm{CuO}$ nanosized particles are similar, their corresponding work function would have changed from the bulk values $[31,32]$ by the same factor. Figure 8 displays the plot of rate of change in the absorbance of $\mathrm{MB}$ for $1 \mathrm{~min}$ in the reaction mixture with catalyst work function, considering only the bulk value.
It can be concluded that as the work function of the particles decreases there occurs an increase in the rate of catalysed reaction, resulting in a decrease in energy barrier for the reaction to proceed and hence the catalyst will be more active. These results are not limited to particular metal oxide catalysts and underline the importance of electronic factor in heterogeneous catalysis by establishing the relationship between catalyst work function and its performance. This observation is quite consistent with our previous two studies in case of metal particles [27,29]. The plausible catalysis mechanism can be proposed on the basis of an electron relay effect $[33,34]$ and is presented graphically in figure 9 .

Concomitant adsorptions of the reactants occur at the catalyst surface with transfer of electron from the electron-rich $\mathrm{BH}_{4}^{-}$species to the catalyst. The metal oxide nanocatalyst further furnishes the electron to $\mathrm{MB}$ and de-adsorption of the product takes place from nanocatalyst surface. Reusability plot of the metal oxide nanocatalysts was not incorporated as it was not reproducible vividly due to subsequent loss of particles during multiple separations, centrifugation and washing processes involved during the recycling steps. Thus, this work offers direct evidence for the interplay of catalyst work function in metal-oxide-NP-catalysed reaction. At least as important, our work provides a fascinating green aqueous approach towards coherent designing of pure metal oxide nanocatalysts and optical establishment of catalyst work function as one of the key parameters for particle-catalysed reactions.

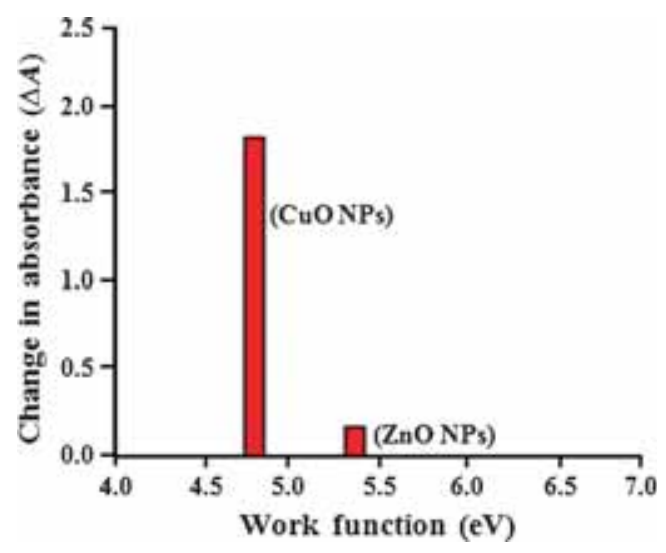

Figure 8. Plot of work function vs. change in absorbance for catalysed reaction after $1 \mathrm{~min}$ at $25^{\circ} \mathrm{C}$.

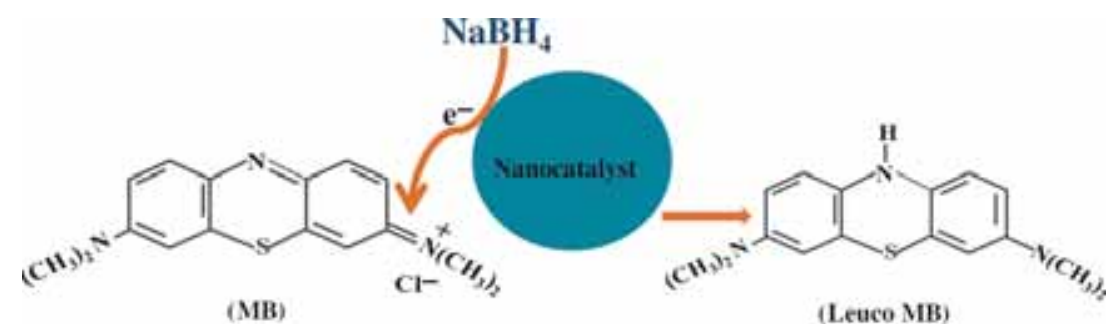

Figure 9. Graphical representation of probable mechanism for NP-catalysed reaction. 


\section{Conclusions}

We have demonstrated a beneficial, straightforward application of green chemistry principles in the preparation of nanoscale $\mathrm{ZnO}$ and $\mathrm{CuO}$ particles utilizing $C$. asiatica plant extract. Such green synthetic strategy is rapidly replacing traditional chemical syntheses. The tailored spherical metal oxide NPs are crystalline and have identical average diameter of about $7 \mathrm{~nm}$. Polyphenols and protein (or amino acids) constituents of the plant materials are believed to be the surface active molecules that stabilized the nanoscopic particles. Our report shows promise of metal oxide NPs in diminution of organic pollutant. Higher catalytic activity of $\mathrm{CuO}$ NPs than $\mathrm{ZnO}$ NPs in the reduction of MB using $\mathrm{NaBH}_{4}$ is attributed to its lesser work function value. This work demonstrates optically the dependence of the kinetics of reaction on the metal oxide catalyst work function. We hope our efforts would be beneficial in tailoring a catalytic system and better understanding of heterogeneous catalysis.

\section{Acknowledgements}

We are grateful to NEHU (SAIF) Shillong, Department of Physics, Manipur University, USIC and Department of Physics, University of Delhi, for providing instruments used during the characterization of particles.

\section{References}

[1] Salata O V 2004 J. Nanobiotechnol. 21

[2] Iravani S 2011 Green Chem. 132638

[3] Njagi E C, Huang H, Stafford L, Genuino H, Galindo H M, Collins J B, Hoag G E and Sui S L 2011 Langmuir 27264

[4] Singh H P, Gupta N, Sharma S K and Sharma R K 2013 Colloids Surf. A: Physicochem. Eng. Aspects 41643

[5] Singh H P, Mitra S and Sharma R K 2014 RSC Adv. 461028

[6] Tong H, Ouyang S, Bi Y, Umezawa N, Oshikiri M and Ye J 2012 Adv. Mater. 24229

[7] Ghosh M, Biswas K, Sundaresan A and Rao C N R 2006 J. Mater. Chem. 16106

[8] Bhakta G, Sharma R K, Gupta N, Cool S, Nurcombe V and Maitra A 2011 Nanomed.: Nanotech. Bio. Med. 7472

[9] Chakraborty S and Guan H 2010 Dalton Trans. 397427

[10] Cuenya B R 2010 Thin Solid Films 5183127

[11] Shankar S S, Rai A, Ahmad A and Sastry M 2004 J. Colloid. Interface Sci. 275496
[12] Baro M, Nayak P, Baby T T and Ramaprabhu S 2013 J. Mater. Chem. A 1482

[13] Kharissova O V, Dias H V R, Kharisov B I, Perez B O and Perez V M J 2013 Trends Biotechnol. 31240

[14] Nadagouda M N 2012 In Modern aspects of bulk crystal and thin film preparation Kolesnikov N (ed) (Rijeka, Croatia: InTech) p 395

[15] Singh H P, Gupta N and Sharma R K 2014 J. Biomed. Ther. Sci. 134

[16] Logeswari P, Silambarasan S and Abraham J 2013 Sci. Iran. F 201049

[17] Das R K, Borthakur B B and Bora U 2010 Mater. Lett. 641445

[18] Singh S, Singh D R, Banu V S and Avinash N 2014 Ind. Crops Prod. 61115

[19] Okonogi S, Sirithunyalug J and Chen Y $2008 \mathrm{XVI}^{\text {th }}$ International conference on bioencapsulation (Dublin, Ireland) p 1

[20] Sathishkumar P, Sweena R, Wu J J and Anandan S 2011 Chem. Eng. J. 171136

[21] Naika H R, Lingaraju K, Manjunath K, Kumar D, Nagaraju G, Suresh D and Nagabhushana H 2015 J. Taibah Univ. Sci. 97

[22] Nune S K, Chanda N, Shukla R, Katti K, Kulkarui R R, Thilakavanthy S, Mekapothula S, Kannan R and Katti K V 2009 J. Mater. Chem. 192912

[23] Prasad R 2014 J. Nanopart. Article ID 963961 doi: $10.1155 / 2014 / 963961$

[24] Zheng W, Guo F and Qian Y 2005 Adv. Funct. Mater. 15331

[25] Deori K, Gupta D, Saha B, Awasthi S K and Deka S 2013 J. Mater. Chem. A 17091

[26] Wang W, Wang L, Shi H and Liang Y 2012 Cryst. Eng. Comm. 145914

[27] Singh H P, Sharma S, Sharma S K and Sharma R K 2014 RSC Adv. 437816

[28] Gupta N, Singh H P and Sharma R K 2011 J. Mol. Cat. A: Chem. 335248

[29] Vayenas C G, Babelis S and Ladas S 1990 Nature 343625

[30] Zhou L and Zachariah M R 2012 Chem. Phys. Lett. 52552677

[31] Kevin M, Ong W L, Lee G H and Ho G W 2011 Nanotechnology 22235701

[32] Zhao Y M, Li Y H, Jin Y Z, Zhang X P, Hu W B, Ahmad I, McCartney G and Zhu Y Q 2007 J. Phys.: Conf. Ser. 61703

[33] Devi H S, Singh N R, Singh H P and Singh T D 2015 J. Environ. Chem. Eng. 32042

[34] Sahoo R, Dutta S, Pradhan M, Ray C, Roy A, Pal T and Pal A 2014 Dalton Trans. 436677 